\title{
Current-density-functional theory of quantum dots in a magnetic field
}

\author{
M. Ferconi and G. Vignale \\ Institute for Theoretical Physics, University of California, Santa Barbara, California 93106-4030 \\ and Department of Physics and Astronomy, University of Missouri-Columbia, Columbia, Missouri 65211
}

(Received 15 August 1994)

\begin{abstract}
We present a study of ground-state energies and densities of quantum dots in a magnetic field, which takes into account correlation effects through the current-density-functional theory. The method is first tested against exact results for the energy and density of two- and three-electron quantum dots, and it is found to yield an accuracy better than 3\%. Then we extend the study to larger dots and compare the results with available experimental data. The orbital and spin angular momenta of the ground state, and the evolution of the density profile as a function of the magnetic field are calculated. Quantitative evidence of edge reconstruction at high magnetic field is presented.
\end{abstract}

Recent progress in nanofabrication techniques has allowed the realization of microstructures in which electrons in a two-dimensional electron gas are confined to an approximately circular region, of radius varying from several hundreds to several thousand angstroms. At the densities characteristic of these quantum dots $\left(n \sim 10^{11}-10^{12} \mathrm{~cm}^{-2}\right)$, as a consequence of the material that hosts the system, a magnetic field $B$ of the order of few tesla is already "strong," i.e., it has a magnetic length $\lambda=\sqrt{\hbar c / e B}$ of the order of the interelectron distance. For this reason, particular attention has been devoted to the evolution of the ground-state properties as function of an applied magnetic field. Experiments using conductance ${ }^{1}$ and single-electron capacitance ${ }^{2}$ spectroscopy have led to the measurement of the electronic spectrum of the dot, and of its addition energy, namely, the energy necessary to add one electron to the system, as a function of the magnetic field. The occurrence of frequent cusps in these functions has been interpreted in terms of crossings between energy levels characterized by different values of spin and orbital angular momentum. In the strong magnetic-field regime, interesting effects, such as "magic" angular momentum quantum numbers, formation of Wigner molecules, and edge reconstruction, have been theoretically predicted. $^{3,4}$

From the standpoint of the theory, the main obstacle to the determination of the energy level structure of quantum dots is the strong electron-electron interaction-the noninteracting problem being exactly solvable, at least in the approximation of parabolic confinement. ${ }^{5}$ Exact diagonalization studies have been carried out for dots with a small number of electrons $^{6-9}(N \leqslant 8)$, and have yielded a very rich scenario. In the case of larger systems, one has to resort to some approximations in order to carry on the calculations. Approximation schemes such as the constant interaction model, ${ }^{10}$ self-consistent Thomas-Fermi method, ${ }^{11}$ the HartreeFock, ${ }^{4,12}$ or other mean-field approximations, ${ }^{13}$ all have the common feature of not including correlation effects. The importance of the latter is all but negligible when the number of electrons in the dot is less than a few hundred. For the unpolarized two-electron case, correlation effects make up to $\sim 10 \%$ of the energy, ${ }^{7}$ which is quite a sizable quantity. At the same time the corresponding Hartree-Fock density profile is incorrect. Moreover, the magnetic-field values at which changes in the quantum numbers occur are influenced by correlation effects. All the above are compelling reasons for including correlations in any model that wants to describe quantitatively the properties of quantum dots.

A full fledged many-body approach appears to be prohibitively difficult at present, for dots containing more than a few electrons. On the other hand, density-functional theory ${ }^{14}$ (DFT) is known to have been successfully applied to many atomic and molecular systems, and it provides-via the local-density approximation (LDA) - a simple method to approximately include electronic correlations. In the present paper, we employ the generalization of DFT known as current-density-functional theory (CDFT), ${ }^{15}$ which is appropriate to deal with systems in the presence of a magnetic field. We emphasize that this theory had never been previously tested against exact results. Quantum dots with only few electrons have enabled us to conduct such a test.

The CDFT scheme for electronic systems in a magnetic field has been reviewed extensively. ${ }^{15}$ Here we limit ourselves to the essentials of the formulation for quantum dots. We consider circularly symmetric dots, and assume a parabolic confining potential $V(r)=\frac{1}{2} m \omega_{0}^{2} r^{2}$ of frequency $\omega_{0}$, with $m$ the effective electron mass. The vector potential corresponding to the uniform magnetic field in the symmetric gauge is $\mathbf{A}(\mathbf{r})=B / 2(-y, x, 0)$. The ground-state energy $E$, the density $n(\mathbf{r})$, and the paramagnetic current density $\mathbf{j}_{p}(\mathbf{r})$ are all expressed in terms of a set of Kohn-Sham orbitals

$$
\psi_{j l \sigma}(\mathbf{r})=e^{-i l \theta} \phi_{j l \sigma}(r),
$$

which are eigenstates of the $z$ component of the angular momentum $-l$, and satisfy the Kohn-Sham equation

$$
\begin{gathered}
\left\{-\frac{\hbar^{2}}{2 m}\left(\frac{\partial^{2}}{\partial r^{2}}+\frac{1}{r} \frac{\partial}{\partial r}-\frac{l^{2}}{r^{2}}\right)-\frac{e \hbar l}{2 m c} B+\frac{m \Omega^{2}}{2} r^{2}-\frac{e \hbar l}{m c} \frac{A_{\mathrm{xc}}}{r}\right. \\
\left.+V_{H}+V_{\mathrm{xc} \sigma}+g \mu_{B} B \sigma\right\} \phi_{i l \sigma}=\epsilon_{i l \sigma} \phi_{i l \sigma},
\end{gathered}
$$

$\sigma$ being the $z$ component of the spin and $\mu_{B}=e \hbar / 2 m c$ the effective Bohr magneton. The renormalized frequency 

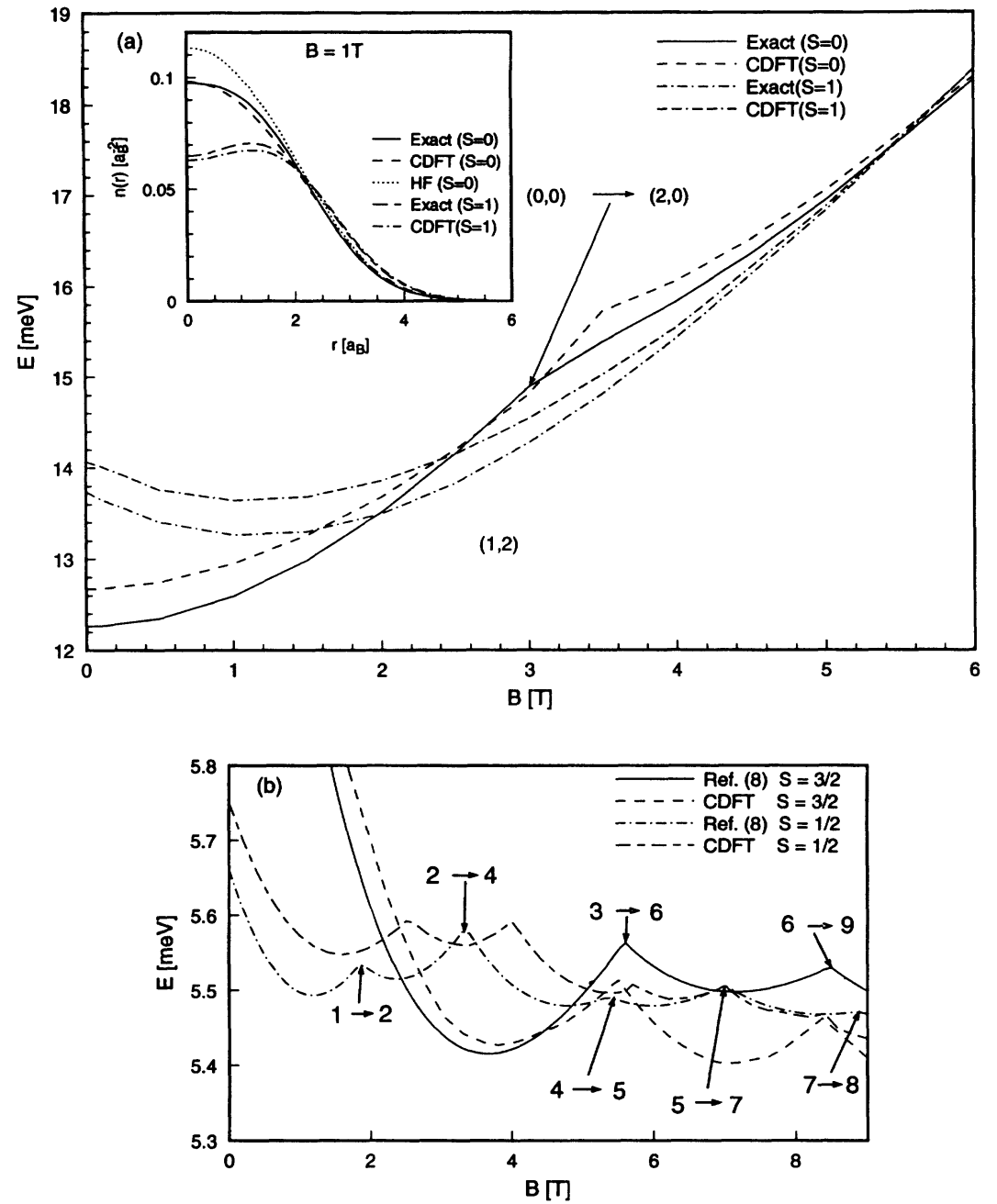

FIG. 1. (a) Ground-state energy for the twoelectron quantum dot, for $S_{z}=0$ and 1 . The transition from $\left(M_{z}, 2 S_{z}\right)=(0,0)$ to $\left(M_{z}, 2 S_{z}\right)=(2,0)$ is marked by an arrow. In the inset the density profiles for the two spin configurations are plotted and compared with the exact and HartreeFock (HF) (Ref. 7) results. (b) Ground-state energy per electron for $S=\frac{3}{2}$ and $\frac{1}{2}$ in a threeelectron quantum dot. The transition points from orbital angular momentum $M_{z 1}$ to $M_{z 2}$ are indicated as $M_{z 1} \rightarrow M_{z 2}$. Parameters appropriate for $\mathrm{GaAs}$ have been chosen, i.e., $m=0.067 m_{e}$, with $m_{e}$ electron mass, $k=12.4$, which give a Bohr radius $a_{B} \sim 98 \AA, \omega_{0}=3.37 \mathrm{meV}$. The Zeeman splitting is neglected.
$\Omega=\sqrt{\omega_{0}^{2}+\omega_{c}^{2} / 4}$, where $\omega_{c}$ is the cyclotron frequency. Explicit expressions for the relevant quantities are

$$
n_{\sigma}(r)=\sum_{\{i l\}}^{N_{\sigma}}\left|\phi_{i l \sigma}(r)\right|^{2}
$$

for the spin density $(\sigma=\{\uparrow, \downarrow\})$,

$$
\mathbf{j}_{p}(r)=j_{p}(r) \hat{\mathbf{e}}_{\theta}=-\frac{\hbar}{m r} \sum_{\{i l \sigma\}}^{N} l\left|\phi_{i l \sigma}(r)\right|^{2} \hat{\mathbf{e}}_{\theta}
$$

for the paramagnetic current density, with $\hat{\mathbf{e}}_{\theta}$ the azimuthal unit vector,

$$
\begin{aligned}
E= & \sum_{\{i l \sigma\}}^{N} \varepsilon_{i l \sigma}-\frac{e^{2}}{2 k} \iint d \mathbf{r} d \mathbf{r}^{\prime} \frac{n(\mathbf{r}) n\left(\mathbf{r}^{\prime}\right)}{\left|\mathbf{r}-\mathbf{r}^{\prime}\right|} \\
& -\sum_{\sigma} \int d \mathbf{r} n_{\sigma}(\mathbf{r}) V_{\mathrm{xc} \sigma}(\mathbf{r})-\frac{e}{c} \int d \mathbf{r} \mathbf{j}_{p}(\mathbf{r}) \cdot \mathbf{A}_{\mathrm{xc}}(\mathbf{r}) \\
& +E_{\mathrm{xc}}[n, \xi, \mathscr{V}]
\end{aligned}
$$

for the energy. In all cases the summation is extended to the $N$ lowest eigenvalues, where $N$ is the number of electrons $\left(N=N_{\uparrow}+N_{\downarrow}\right)$. The Kohn-Sham equation includes the selfconsistent Hartree potential

$$
V_{H}(r)=2 \pi \frac{e^{2}}{k} \int d r^{\prime} r^{\prime} n\left(r^{\prime}\right) /\left|\mathbf{r}-\mathbf{r}^{\prime}\right|
$$

the exchange-correlation potential

$$
V_{\mathrm{xc} \sigma}(\mathbf{r})=\left.\frac{\delta E_{\mathrm{xc}}[n, \xi, \mathscr{V}]}{\delta n_{\sigma}(\mathbf{r})}\right|_{n_{-\sigma}, \mathscr{V}}-\frac{e}{c} \mathbf{A}_{\mathrm{xc}}(\mathbf{r}) \cdot \frac{\mathbf{j}_{p}(\mathbf{r})}{n(\mathbf{r})},
$$

and the exchange-correlation vector potential

$$
\frac{e}{c} \mathbf{A}_{\mathrm{xc}}=\frac{e}{c} A_{\mathrm{xc}} \hat{\mathbf{e}}_{\theta}=\left.\frac{m c}{e n(r)} \frac{\partial}{\partial r}\left(\frac{\delta E_{\mathrm{xc}}[n, \xi, \mathscr{V}]}{\delta \mathscr{V}}\right)\right|_{n, \xi} .
$$

These last two quantities are formally defined as functional derivatives of the exchange-correlation energy functional $E_{\mathrm{xc}}$ - a functional of the density $n$, the spin polarization $\xi=\left(n_{\uparrow}-n_{\downarrow}\right) /\left(n_{\uparrow}+n_{\downarrow}\right)$, and the vorticity $\mathscr{T}(\mathbf{r})=\mathscr{V}(r) \hat{\mathbf{e}}_{z}$ $=-m c / e r \partial\left[r j_{p}(r) / n(r)\right] / \partial r \hat{\mathbf{e}}_{z}$. The exchange-correlation energy has been calculated in the LDA 


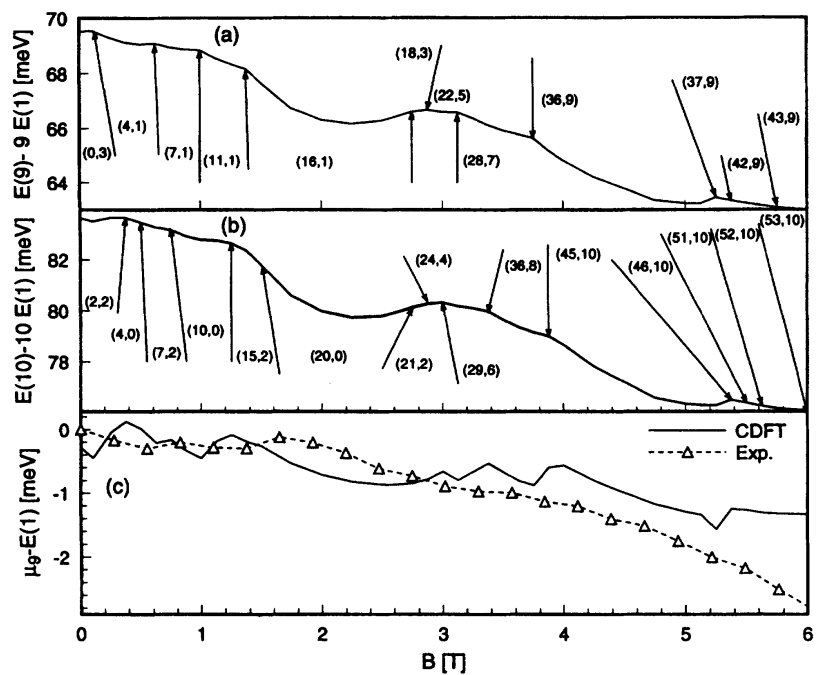

FIG. 2. (a) Magnetic-field dependence of the ground-state energy for the nine-electron dot. (b) Same for the ten-electron dot. The quantum numbers $\left(M_{z}, 2 S_{z}\right)$, of the ground states for the nine and ten electrons are shown, and they refer to the regions of magnetic field between two successive arrows. (c) Magnetic-field dependence of the addition energy for the nine-electron dot. The triangles represent the experimental points (Ref. 2), and the dashed line is a guide to the eye. A $g$ factor of $g=-0.44$, and $\omega_{0}=3.31 \mathrm{meV}$ have been assumed.

$$
E_{\mathrm{xc}}=\int d \mathbf{r} n(r) \epsilon_{\mathrm{xc}}[n(\mathbf{r}), \xi(\mathbf{r}), \mathscr{V}(\mathbf{r})]
$$

where $\epsilon_{\mathrm{xc}}$ is the exchange-correlation energy density of the uniform two-dimensional electron gas in an effective magnetic field $\mathscr{V}(\mathbf{r})$ which, in the LDA is taken to be approximately equal to the external magnetic field B. Following Rasolt and Perrot ${ }^{16}$ we have chosen

$$
\epsilon_{\mathrm{xc}}[n, \xi, \mathscr{V}]=\left(\epsilon_{\mathrm{xc}}^{\mathrm{LWM}}[n, \nu]+\nu^{4} \epsilon_{\mathrm{xc}}^{\mathrm{TC}}[n, \xi]\right) /\left(1+\nu^{4}\right),
$$

where $\nu=2 \pi \lambda^{2} n$ is the filling factor. The expression connects the fitted form of Levesque, Weis, and MacDonald ${ }^{17}$ $\epsilon_{\mathrm{xc}}^{\mathrm{LWM}}[n, \nu]$, which is valid for small filling factor (or large magnetic field), to the form given by Tanatar and Ceperley ${ }^{18}$ $\epsilon_{\mathrm{xc}}^{\mathrm{TC}}[n, \xi]$ for zero magnetic field. Different Padé polynomials that reproduce the known limiting cases do not change appreciably the general behavior in the regions where they contribute to the energy. It must be underlined that, in contrast to previous approaches, the present method does not use a truncated basis set of Landau or Fock-Darwin orbitals as a basis in which to expand the solution of the Kohn-Sham equation. For this reason, our approach is completely unbiased with respect to the strength of the magnetic field and should work equally well, a priori, for weak and strong magnetic fields.

The accuracy of our method has been tested in the twoand three-electron cases, where exact results are available. This is a very severe test for any uniform electron-gas-based energy functional. Our results are summarized in Fig. 1. For two electrons, we plot the ground-state energies of the $S=0$ and 1 symmetry states versus the magnetic field. It is seen that the deviation from the exact energy is contained to
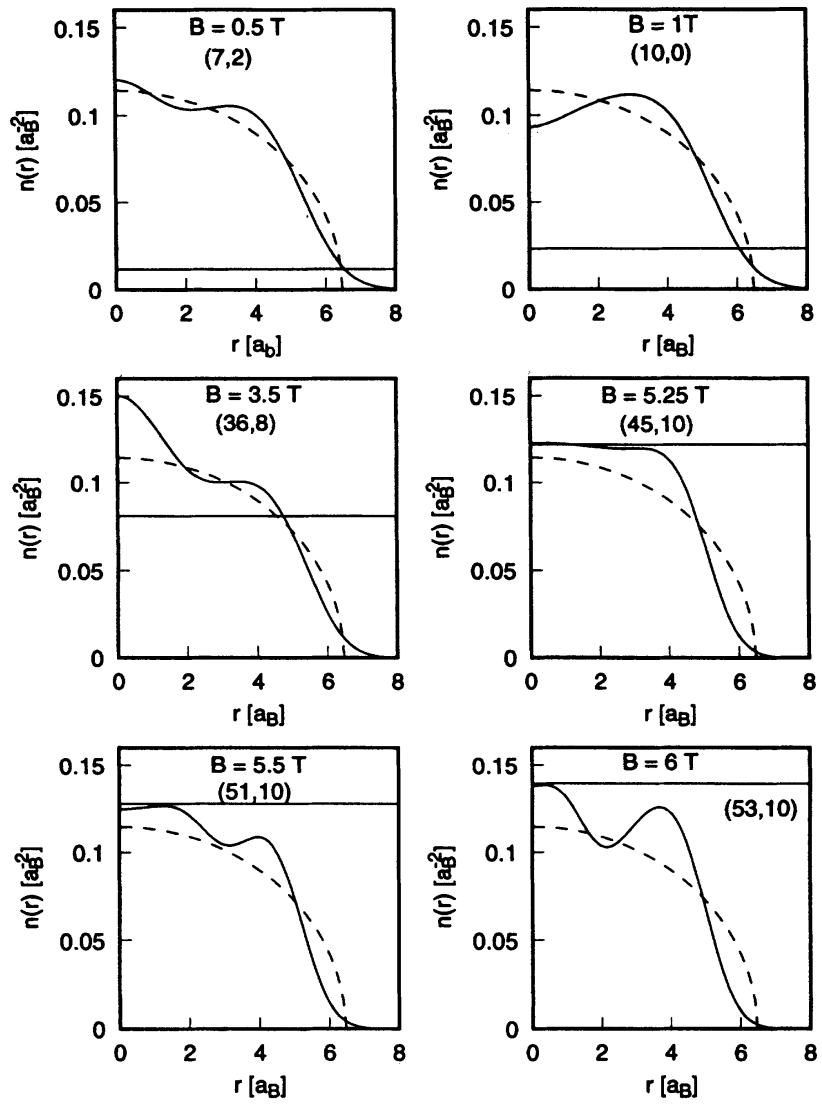

FIG. 3. Evolution of the density profile with the magnetic field for the ten-electron quantum dot of Fig. 2. The classical distribution (Ref. 13) is given by the dashed line, while the density corresponding to filling factor $\nu=1=1 / 2 \pi \lambda^{2}$ is represented by the horizontal line. The quantum numbers $\left(M_{z}, 2 S_{z}\right)$ are also shown.

$\$ 3 \%$. A remarkable improvement is achieved in the $S=0$ case upon the Hartree-Fock calculation, ${ }^{7}$ which gives an error of $\sim 10 \%$. Another satisfying feature is that the orbital angular momentum-which is known to increase in steps with increasing magnetic field-is correctly obtained, apart from a slight difference in the value of the magnetic field at which the discontinuities occur. Excellent agreement is also obtained in the ground-state density distributions for each spin symmetry state [see inset of Fig. 1(a)], which again shows considerable improvement upon the Hartree-Fock result for the singlet. With increasing magnetic field, the density distribution moves inward and becomes more localized about the origin, as long as the angular momentum remains zero. This leads to an increase in electrostatic energy which is suddenly released when the angular momentum jumps to a finite value, and the density peak moves outward to a finite radius. This pattern is repeated every time that the orbital angular momentum increases. The inward displacement of the density at constant angular momentum is followed by a sudden outward expansion when the angular momentum increases.

In the three-electron case, ${ }^{8}$ we have plotted in Fig. 1(b) the ground-state energies per particle of the $S=\frac{3}{2}$ and $\frac{1}{2}$ ground states. An energy of $\hbar \Omega$ per particle has been subtracted in order to show more clearly the rich structure of the 
ground-state energy, featuring several changes in orbital angular momentum. Even for this small part of the total energy, CDFT is able to correctly reproduce the exact behavior, with an error that even in the worst situation is below $\sim 5 \%$, being much lower in general. As in the two-electron case, we note a loss of accuracy for very high magnetic field, due to the increase in the number of quasidegenerate Kohn-Sham orbitals. We also notice that the exchange-correlation vector potential $A_{\text {xc }}$ gives a very small contribution to the energy.

The above results provide convincing evidence that CDFT is a powerful tool to predict qualitatively-and quantitatively, within an accuracy of few percent-the evolution of the ground-state properties of quantum dots with magnetic field. We next apply it to the calculation of quantum dots with a larger number of electrons. Figure 2 shows the ground-state energies and orbital symmetries of nine- and ten-electron quantum dots, as well as the addition energy for the nine-electron system. A comparison of the results with the experimental data ${ }^{2}$ is shown in the lowest panel of Fig. 2 . Following Hawrylak ${ }^{19}$ we have assumed a dependence of the effective confining potential $\omega_{N}$ on the number of electrons $N$ in the dot, of the form $\omega_{N}=\sqrt{\omega_{0}^{2}+e^{2} N / m k d^{3}}$, where $d$ is the distance of the positive charge background from the plane of the dot, and $\omega_{0}$ is the "empty dot" confining frequency. A vertical rigid shift of the theoretical curve has also been performed on the basis that there is a constant term for the energy at a fixed number of electrons in the dot, depending on the geometry of the environment in which the system is created and on the probe that measures the addition energies. Although the patterns of cusps in the theoretical and experimental curves appear to be very different, we suggest that the discrepancy may be attributed, in large part, to a systematic underestimation of the values of the magnetic field at which the quantum number transitions occur. In other words, a rigid shift of the theoretical curve to higher magnetic field would considerably improve the agreement with experiment. In general, however, the observed difference suggests that the conventional model of a confinement essentially independent on $N$ and $B$ may not be an adequate rep- resentation of reality, at the level of accuracy considered here.

As a final point of interest, we have studied the evolution of the density profile as a function of magnetic field, and some results for the ten-electron dot are presented in Fig. 3. According to the classical electrostatic analysis, ${ }^{13}$ the distribution should look like the dome shown in Fig. 3, which is magnetic-field independent, with small corrections induced by the quantized kinetic energy. While at low values of the magnetic field the electrostatic behavior is reasonably reproduced, apart from oscillations, with increasing magnetic field significant changes take place, reflecting the different values of quantum numbers. At $B \simeq 3.9 \mathrm{~T}$ the ten-electron dot becomes fully spin polarized, and its orbital angular momentum $M_{z}=45$ corresponds to the "maximum density droplet," i.e., all the orbitals with $l=0,1, \ldots, 9$ are occupied by a spin-up electron. ${ }^{20}$ As the magnetic field is further increased, some electrons are promoted to orbitals with $l>9$ leaving some of the orbitals with $l<9$ empty. In terms of the density profile, this effect appears as an edge reconstruction, similar to the one discussed in Ref. 4 , whereby the charge density first decreases and then increases in the edge region, before falling to zero. The physical reason is that the exchangecorrelation energy favors a higher density in the edge region, while the electrostatic energy, of course, opposes it. The precise size and shape of the edge is determined by the competition of these two energies. As can be seen, this phenomenon is not restricted to large dots, but occurs even in small systems. More details on this problem will be published elsewhere.

We thank J. Perdew for providing us an atomic densityfunctional code from which we have derived ours, $R$. Ashoori for giving us his experimental data, and J. Palacios for sending us a copy of his work prior to publication. We acknowledge useful conversations with Pawel Hawrylak, Ray Ashoori, Eberhard Gross, and Francois Perrot. We acknowledge support from NSF Grants Nos. DMR 9100988 and DMR 9403908 and hospitality at the ITP where part of this work was done, under NSF Grant No. PHY89-04035.
${ }^{1}$ P. L. McEuen et al., Phys. Rev. Lett. 66, 1926 (1991).

${ }^{2}$ R. C. Ashoori et al., Phys. Rev. Lett. 68, 3088 (1992); 71, 613 (1993).

${ }^{3}$ P. A. Maksym, Physica B 184, 385 (1993).

${ }^{4}$ C. de C. Chamon and X. G. Wen, Phys. Rev. B 49, 8227 (1994).

${ }^{5}$ V. Fock, Z. Phys. 47, 446 (1928); C. G. Darwin, Proc. Philos. Soc. London 27, 86 (1930).

${ }^{6}$ P. A. Maksym and T. Chakraborty, Phys. Rev. Lett. 65, 108 (1990); Phys. Rev. B 45, 1947 (1992).

${ }^{7}$ D. Pfannkuche, V. Gudmundsson, and P. A. Maksym, Phys. Rev. B 47, 2244 (1993).

${ }^{8}$ P. Hawrylak and D. Pfannkuche, Phys. Rev. Lett. 70, 485 (1993).

${ }^{9}$ S.-R. E. Yang, A. H. MacDonald, and M. D. Johnson, Phys. Rev. Lett. 71, 3194 (1993).

${ }^{10}$ C. W. J. Beenakker, H. van Houten, and A. A. M. Staring, Phys.
Rev. B 44, 1657 (1991).

${ }^{11}$ P. L. McEuen et al., Phys. Rev. B 45, 11419 (1992).

${ }^{12}$ J. J. Palacios et al., Phys. Rev. B 50, 5760 (1994).

${ }^{13}$ M. M. Fogler, E. I. Levin, and B. I. Shklovskii, Phys. Rev. B 49, 13767 (1994).

${ }^{14}$ For a recent review, see R. M. Dreizler and E. K. U. Gross, Density Functional Theory. An Approach to the Quantum ManyBody Problem (Springer-Verlag, Berlin, 1990).

${ }^{15}$ G. Vignale and M. Rasolt, Phys. Rev. Lett. 59, 2360 (1987); Phys. Rev. B 37, 10685 (1988).

${ }^{16}$ M. Rasolt and F. Perrot, Phys. Rev. Lett. 69, 2563 (1992).

${ }^{17}$ D. Levesque, J. J. Weis, and A. H. MacDonald, Phys. Rev. B 30, 1056 (1984).

${ }^{18}$ B. Tanatar and D. M. Ceperley, Phys. Rev. B 39, 5005 (1989).

${ }^{19}$ P. Hawrylak, Phys. Rev. Lett. 71, 3347 (1993).

${ }^{20}$ A. H. MacDonald et al., Aust. J. Phys. 46, 345 (1993). 